\title{
Quasirelativistic energy-consistent 5f-in-core pseudopotentials for trivalent actinide elements
}

\author{
Anna Moritz • Xiaoyan Cao • Michael Dolg
}

Received: 1 August 2006 / Accepted: 1 September 2006 / Published online: 21 November 2006

(C) Springer-Verlag 2006

\begin{abstract}
Quasirelativistic energy-consistent 5f-in-core pseudopotentials modelling trivalent actinides, corresponding to a near-integral $5 \mathrm{f}^{n}$ occupation $(n=0-14$ for Ac-Lr), have been generated. Energy-optimized (6s5p4d), (7s6p5d), and (8s7p6d) primitive valence basis sets contracted to polarized double to quadruple zeta quality as well as $2 \mathrm{f1} \mathrm{g}$ correlation functions have been derived. Corresponding smaller basis sets $(4 \mathrm{~s} 4 \mathrm{p} 3 \mathrm{~d})$, (5s5p4d), and (6s6p5d) suitable for calculations on actinide(III) ions in crystalline solids form subsets of these basis sets designed for calculations on neutral molecules. Results of Hartree-Fock test calculations for actinide(III) monohydrates and actinide trifluorides show a satisfactory agreement with corresponding calculations using $5 f$-in-valence pseudopotentials. Even in the beginning of the actinide series, where the $5 \mathrm{f}$ shell is relatively diffuse, only quite acceptable small deviations occur as long as the 5 f-shell does not participate significantly in covalent bonding.
\end{abstract}

Keywords Actinides · Pseudopotentials · Valence basis sets · Actinide(III) monohydrates $\cdot$ Actinide trifluorides

Electronic supplementary material Supplementary material is available in the online version of this article at http://dx.doi.org/10.1007/s00214-006-0180-7 and is accessible for authorized users.

A. Moritz $\cdot$ X. Cao $\cdot$ M. Dolg $(\bowtie)$

Institut für Theoretische Chemie,

Universität zu Köln, Greinstr. 4,

50939 Köln, Germany

e-mail: m.dolg@uni-koeln.de

\section{Introduction}

All actinide elements are radioactive and some of them, namely uranium and plutonium, have crucial importance as nuclear power sources, e.g. for the production of electricity as well as for the development of nuclear weapons [1]. In addition actinides can be potentially applied in radiotherapy. In all cases the radioactive nuclear waste management risks must be addressed sooner or later [2]. Consequently, a detailed understanding of actinide properties is highly desirable. However, actinide chemistry is still a considerable challenge for both experimental and theoretical work. From the experimental point of view the limited availability of most actinide elements and their severe radioactivity are the main obstacles. For quantum chemical first-principle studies of systems containing actinide elements, the main challenge arises from the significant contributions of relativity as well as electron correlation [3-6].

Among the approaches developed in relativistic quantum chemistry, the method of ab initio pseudopotentials (PPs) is one of the most successful ones. The explicit calculations are restricted to the chemically relevant valence electron system and relativistic effects are only implicitly accounted for by a proper adjustment of free parameters in the valence model Hamiltonian. While the first aspect leads to a reduction of the computational effort, the second allows the inclusion of scalarrelativistic contributions in a nonrelativistic framework. For f-elements several energy-consistent PPs with different core definitions, i.e. $4 \mathrm{f}$-in-core $[7,8]$ as well as $4 \mathrm{f}$ in-valence PPs $[9,10]$ for lanthanides and $5 \mathrm{f}$-in-valence PPs $[11,12]$ for actinides, were published and have been successfully applied by many researchers [6]. However, due to the well-known occasional stronger involvement 
of actinide $5 \mathrm{f}$ orbitals in chemical bonding the development of 5f-in-core actinide PPs was not attempted so far to our knowledge.

An additional complexity for quantum mechanical calculations arises from the possible presence of several open shells with different main and angular quantum numbers, i.e. in the neutral actinides and their ions often $5 \mathrm{f}$ as well as $6 \mathrm{~d}, 7 \mathrm{~s}$, and possibly $7 \mathrm{p}$ orbitals may be partially occupied in the ground state or low-lying excited states. A ${ }^{2 S+1} L_{J}$ term arising from a $5 \mathrm{f}^{n}$ subshell may lead to a spin $S$ as large as 7/2 and an angular momentum $L$ as large as 12 resulting in a great number of lowlying $L S$ states. Due to the coupling between the $5 \mathrm{f}^{n}$ subshell to other partially occupied $6 \mathrm{~d}$, $7 \mathrm{~s}$, or $7 \mathrm{p}$ shells even more extreme values of $S$ and $L$ may result [13]. Therefore calculations on molecules containing actinide atoms/ions using the available 5f-in-valence PPs [11] or an all-electron (AE) approach are often computationally very demanding or even not feasible. In the case of the lanthanides similar difficulties could be efficiently handled by attributing the open but rather core-like 4f shell to the PP core $[7,8]$. Instead of a large number of individual electronic states which give rise to almost identical spectroscopic parameters (e.g. geometries, vibrational frequencies) one deals with an average of states belonging to a so-called superconfiguration [14, 15]. Using this approximation even calculations on large metal-organic complexes of medical interest (X-ray radiation therapy, photodynamic therapy, magnetic resonance imaging), e.g. lanthanide(III) texaphyrins, became possible $[15,16]$. In analogy to these 4 f-in-core lanthanide PPs we thus decided to generate corresponding 5f-in-core actinide PPs and to explore their range of applicability. The $1 \mathrm{~s}-5 \mathrm{f}$ shells are included in the PP core, while only the $6 \mathrm{~s}, 6 \mathrm{p}, 6 \mathrm{~d}$, and $7 \mathrm{~s}$ orbitals of the actinide elements are considered as valence orbitals.

From a chemical point of view actinide elements in particular late actinides (Am-Lr) and actinium are usually trivalent [1] corresponding to the presence of $5 \mathrm{f}^{n}$ ( $n=0-14$ for Ac-Lr) subconfigurations. Some early actinides can reach higher valencies up to seven, since in the beginning of the actinide series the $5 \mathrm{f}$ shell is considerably diffuse and can easily participate in chemical bonding. Nevertheless, we restrict our present study to $5 \mathrm{f}$-in-core PPs corresponding to trivalent $\left(5 \mathrm{f}^{n}\right.$ occupation) actinide atoms ( $n=0-14$ for Ac-Lr) and the various valence basis sets for use in molecular calculations. PPs corresponding to other valencies, e.g. divalency $\left(5 \mathrm{f}^{n+1}, n=5-13\right.$ for $\left.\mathrm{Pu}-\mathrm{No}\right)$ and tetravalency $\left(5 \mathrm{f}^{n-1}, n=1-9\right.$ for Th-Cf), have also been generated together with corresponding valence basis sets, but have not been thoroughly tested in molecular applications so far. Results of Hartree-Fock (HF) calculations using the generated PPs for actinide(III) monohydrates $\mathrm{An}^{3+}$ $\mathrm{H}_{2} \mathrm{O}$ and actinide trifluorides $\mathrm{AnF}_{3}(\mathrm{An}=\mathrm{Ac}-\mathrm{Lr})$ are compared mainly to corresponding calculations using 5f-in-valence PPs, since only very little experimental data as well as AE results from other authors are available.

In preliminary studies of actinide(III) motexafin complexes $\left(\mathrm{An}-\mathrm{Motex}^{2+}, \mathrm{An}=\mathrm{Ac}, \mathrm{Cm}, \mathrm{Lr}\right)$ at the level of gradient corrected hybrid density functional theory (DFT/B3LYP), the 5f-in-core approach already performed encouragingly well [17]. A similar statement holds for yet unpublished studies of the hydration behaviour of trivalent actinide ions [18]. Moreover, unpublished $\operatorname{CCSD}(\mathrm{T})$ results of Cao for the vibrational frequencies of $\mathrm{UF}_{3}$ obtained with the $5 \mathrm{f}$-in-core approach are within the experimental error bars. Despite the widespread common knowledge that the actinide $5 \mathrm{f}$ shell is chemically active and cannot be attributed to the core, we found ample quantitative evidence that such an approximation can be made without too large loss of accuracy for many cases, thus making a considerable part of computational actinide chemistry significantly more easy. We expect that the rather crude approximation proposed here will at least work well for the heavier actinides where the $5 \mathrm{f}$ shell is sufficiently core-like, both from a geometric and an energetic point of view [5].

\section{Method}

The method of relativistic energy-consistent ab initio pseudopotentials is described in detail elsewhere [7,9, 11] and will be outlined here only briefly. The valenceonly model Hamiltonian for a system with $n$ valence electrons and $N$ nuclei with charges $Q$ is given as

$H_{v}=-\frac{1}{2} \sum_{i}^{n} \Delta_{i}+\sum_{i<j}^{n} \frac{1}{r_{i j}}+\sum_{i}^{n} \sum_{I}^{N} V_{I}\left(r_{i}\right)+\sum_{I<J}^{N} \frac{Q_{I} Q_{J}}{R_{I J}}$.

Here $i$ and $j$ are electron indices, $I$ and $J$ are nuclear indices. $V_{I}\left(r_{i}\right)$ denotes a semilocal effective core potential (ECP) for nucleus $I$

$V_{I}\left(r_{i}\right)=-\frac{Q_{I}}{r_{I i}}+\sum_{l} \sum_{k} A_{l k}^{I} \exp \left(-a_{l k}^{I} r_{I i}^{2}\right) P_{l}^{I}$.

$P_{l}^{I}$ is the projection operator onto the Hilbert subspace of nucleus $I$ with angular momentum $l$

$P_{l}^{I}=\sum_{m_{l}}\left|l m_{l}\right\rangle\left\langle l m_{l}\right|$ 
The free parameters $A_{l k}^{I}$ and $a_{l k}^{I}$ were adjusted by a least-squares fit to the total valence energies of a multitude of low-lying electronic states of the neutral atom and its cations. The reference data needed for the construction of the pseudopotentials were taken from relativistic AE calculations using the so-called WoodBoring (WB) scalar-relativistic HF approach. Both AE $\mathrm{WB}$ as well as PP calculations were performed with an atomic finite-difference HF scheme [19] in order to avoid basis set effects in the determination of the PP parameters. The 5f-in-core PPs for actinides are 11 valence electron PPs corresponding to a trivalent situation ( $5 \mathrm{f}^{n}$ subconfiguration with $n=0-14$ for Ac-Lr), i.e. the $1 \mathrm{~s}-5 \mathrm{f}$ shells are included in the PP core, while all other shells with main quantum number larger than five are treated explicitly. The s-, p-, and d-PPs which are composed of two Gaussians each ( $k=2$ in (2), i.e. 12 parameters) were adjusted to the total valence energies of 14 reference configurations. In order to allow for some participation of the $5 \mathrm{f}$ orbitals in chemical bonding the f-parts of the PPs are designed to describe partial occupations of the $5 \mathrm{f}$ shell, i.e. the $5 \mathrm{f}$ occupation numbers $q$ modelled with the PPs might be $n \leq q<n+1$ for trivalent actinide atoms in molecules. In slight variation to earlier work $[7,8]$ the f-PPs consist of two types of potentials $V_{1}$ and $V_{2}$ which are linear combined as follows

$V=\left(1-\frac{n}{14}\right) V_{1}+\frac{n}{14} V_{2}$.

Here $n$ is the number of electrons in the $5 \mathrm{f}$ orbitals. $V_{1}$ and $V_{2}$ model $5 \mathrm{f}$ shells which can and respectively cannot accommodate an additional electron. $V_{1}$ is the exact potential for Ac $\left(5 \mathrm{f}^{0}, n=0\right)$, whereas $V_{2}$ is exact for $\operatorname{Lr}\left(5 \mathrm{f}^{14}, n=14\right) . V_{1}$ was adjusted to the four reference configurations $\mathrm{An}^{10+} 5 \mathrm{f}^{n+1}, 5 \mathrm{f}^{n} 6 \mathrm{f}^{1}, 5 \mathrm{f}^{n} 7 \mathrm{f}^{1}$, and $5 \mathrm{f}^{n} 8 \mathrm{f}^{1}$, whereas $V_{2}$ was adjusted to the three reference configurations $\mathrm{An}^{10+} 5 \mathrm{f}^{n} 6 \mathrm{f}^{1}, 5 \mathrm{f}^{n} 7 \mathrm{f}^{1}$, and $5 \mathrm{f}^{n} 8 \mathrm{f}^{1}$. Thus, the f-PPs allow for a decreasing participation of the $5 \mathrm{f}$ orbitals in chemical bonding along the actinide series, i.e. the $5 \mathrm{f}$ shell can completely, partially, and not at all contribute to chemical bonding for Ac, Th-No, and Lr, respectively. The errors in the total valence energies of finite-difference HF calculations are smaller than 0.1 and $0.15 \mathrm{eV}$ for s-, p-, d-PPs and f-PPs, respectively.

The Gaussian type orbital (GTO) valence basis sets for the actinide $5 \mathrm{f}$-in-core PPs were generated in a similar manner as those for the lanthanide 4f-in-core PPs [20]. In order to compare the basis set effects, three different sets of primitive Gaussian functions (4s $4 \mathrm{p} 3 \mathrm{~d}$ ), (5s5p4d), and (6s6p5d) were derived. First, these three sets of exponents for use in crystal calculations were HF energy-optimized [21] for the $6 s^{2} 6 p^{6} 6 d^{1}$ valence subcon- figuration of doubly charged actinide cations. To avoid linear dependency in solid-state calculations which are usually caused by overlap between too diffuse functions of the densely packed atoms all exponents, which became smaller than 0.15 , were fixed to this value and the remaining exponents were reoptimized. Furthermore, all optimizations were carried out with the requirement that the ratio of exponents in the same angular symmetry must be at least 1.5 because in particular steep Gaussians tend to a coalescence resulting in a linearly dependent basis [22]. The basis set errors in the valence energies are smaller than $0.26,0.13$, and $0.12 \mathrm{eV}$ for $(4 \mathrm{~s} 4 \mathrm{p} 3 \mathrm{~d}),(5 \mathrm{~s} 5 \mathrm{p} 4 \mathrm{~d})$, and $(6 \mathrm{~s} 6 \mathrm{p} 5 \mathrm{~d})$, respectively. Secondly, the valence basis sets were augmented by adding two $\mathrm{s}$, one $\mathrm{p}$, and one d low-exponent Gaussian functions yielding $(6 \mathrm{~s} 5 \mathrm{p} 4 \mathrm{~d}),(7 \mathrm{~s} 6 \mathrm{p} 5 \mathrm{~d})$, and $(8 \mathrm{~s} 7 \mathrm{p} 6 \mathrm{~d})$ primitive sets for use in molecular calculations. The added exponents were HF energy-optimized [21] for the $6 d^{1} 7 s^{2}$ valence subconfiguration of the neutral atoms. The differences in the valence energies are at most 0.17 , 0.09 , and $0.08 \mathrm{eV}$ for $(6 \mathrm{~s} 5 \mathrm{p} 4 \mathrm{~d}),(7 \mathrm{~s} 6 \mathrm{p} 5 \mathrm{~d})$, and $(8 \mathrm{~s} 7 \mathrm{p} 6 \mathrm{~d})$, respectively. Thirdly, the basis sets were contracted using different segmented contraction schemes to yield basis sets of approximately valence double, triple, and quadruple zeta quality for the $s$ and $p$ symmetries. In case of d symmetry at least a triple zeta contraction was necessary and additional sets with less tight $\mathrm{d}$ contraction are also offered (VDZ: [4s3p3d], VTZ: [5s4p3d], [5s4p4d], and VQZ: [6s5p4d], [6s5p5d]). The errors of all contracted basis sets are below $0.2 \mathrm{eV}$ and those of VTZ as well as VQZ contractions of (7s6p5d) and (8s7p6d) are at most $0.1 \mathrm{eV}$. Fourthly, sets of $2 \mathrm{f} 1 \mathrm{~g}$ correlation/polarization functions were energy-optimized in configuration interaction (CI) calculations [23] for the $6 \mathrm{~d}^{1} 7 \mathrm{~s}^{2}$ configuration of Ac and Lr. The $2 \mathrm{f} 1 \mathrm{~g}$ exponents for the actinides Th-No were interpolated. The parameters of PPs and basis sets are compiled in the electronic supplementary material of this publication. They are also available from the authors and will be incorporated, e.g. into the MOLPRO [23] basis set library [24].

The test calculations for $\mathrm{An}^{3+}-\mathrm{H}_{2} \mathrm{O}(\mathrm{An}=\mathrm{Ac}-\mathrm{Lr})$ were carried out with MOLPRO [23] using the newly developed 5f-in-core LPPs (large-core PP with 11 valence electrons and 78-92 core electrons for Ac-Lr) as well as 5f-in-valence SPPs [11] (small-core PP with 60 core electrons and 29-43 valence electrons for Ac-Lr). For H and O Dunning's (4s1p) and (9s4p1d) cc-pVDZ (correlation-consistent polarized VDZ) basis sets [25] were used. In order to study the basis set effects, we also applied $(6 s 3 p) /[4 s 3 p]$ and $(11 s 6 p 3 d) /[5 s 4 p 3 d]$ augcc-pVTZ (augmented correlation-consistent polarized VTZ) as well as $(7 \mathrm{~s} 4 \mathrm{p} 3 \mathrm{~d}) /[5 \mathrm{~s} 4 \mathrm{p} 3 \mathrm{~d}]$ and $(13 \mathrm{~s} 7 \mathrm{p} 4 \mathrm{~d} 3 \mathrm{f}) /$ [6s5p4d3f] aug-cc-pVQZ basis sets $[25,26]$ for $\mathrm{H}$ and 
$\mathrm{O}$, respectively. The LPP calculations were carried out choosing (8s7p6d2f) valence basis sets for An which were augmented by one $\mathrm{g}$ function in the case of aug-cc$\mathrm{pVQZ}$ basis sets for $\mathrm{H}$ and $\mathrm{O}$. The SPP calculations were performed using generalized contracted (14s13p10d8f)/ [6s6p5d4f] basis sets [12] which were augmented by $(6 \mathrm{~g}) /[3 \mathrm{~g}]$ diffuse functions in the case of aug-cc-pVQZ basis sets for $\mathrm{H}$ and $\mathrm{O}$. The geometry optimization of $\mathrm{An}^{3+}-\mathrm{H}_{2} \mathrm{O}(\mathrm{An}=\mathrm{Ac}-\mathrm{Lr})$ was realized in $C_{2 v}$ symmetry using $\mathrm{HF}$ and state-averaged multiconfiguration selfconsistent field (MCSCF) for LPP and SPP, respectively. The state-averaging was necessary to avoid symmetrybreaking at the orbital level, since the program MOLPRO [23] is limited to the $D_{2 h}$ point group and subgroups. The actinide-water binding energy was obtained by $E_{\text {bond }}=E\left(\mathrm{An}^{3+}\right)+E\left(\mathrm{H}_{2} \mathrm{O}\right)-E\left(\mathrm{An}^{3+}-\mathrm{H}_{2} \mathrm{O}\right)$, where the actinide ion was assumed to be in the same $5 \mathrm{f}^{n}$ configuration as in the complex.

The results for $\mathrm{AnF}_{3}(\mathrm{An}=\mathrm{Ac}-\mathrm{Lr})$ were also determined by LPP/HF and SPP/state-averaged MCSCF calculations [23]. For F Dunning's aug-cc-pVQZ basis set [25] was applied and for An segmented contracted (7s6p5d2f1g)/[5s4p3d2f1g] and (14s13p10d8f6g)/ [10s9p5d4f3g] [27] valence basis sets were used for LPP and SPP calculations, respectively. The geometries were optimized imposing $\mathrm{C}_{3 v}$ symmetry and the actinidefluorine binding energy was calculated by $E_{\text {bond }}=$ $\left[E(\mathrm{An})+3 \times E(\mathrm{~F})-E\left(\mathrm{AnF}_{3}\right)\right] / 3$, where the actinide atom was assumed to be in the lowest state of the $5 \mathrm{f}^{n}$ $6 d^{1} 7 s^{2}$ configuration. Since the actual symmetry used in the calculations with MOLPRO [23] is $\mathrm{C}_{s}$, state-averaging was applied to avoid symmetry breaking.

\section{Results and discussion}

The results for some properties (namely the bond length $R_{e}$ and the binding energy $\left.E_{\text {bond }}\right)$ of $\mathrm{An}^{3+}-\mathrm{H}_{2} \mathrm{O}$ $(\mathrm{An}=\mathrm{Ac}-\mathrm{Lr})$ as well as of $\mathrm{AnF}_{3}(\mathrm{An}=\mathrm{Ac}-\mathrm{Lr})$ will be presented here in order to demonstrate the transferability of both the 5f-in-core PPs and the corresponding basis sets to a molecular environment. Moreover, Mulliken orbital populations of these compounds will be discussed.

\subsection{Actinide(III) monohydrates}

The HF calculations for $\mathrm{An}^{3+}-\mathrm{H}_{2} \mathrm{O}(\mathrm{An}=\mathrm{Ac}-\mathrm{Lr})$ using LPPs will be compared to those using SPPs as well as to AE Dirac-Hartree-Fock (DHF) calculations [28]. AE data are only available for five actinide(III) monohydrates $\mathrm{An}^{3+}-\mathrm{H}_{2} \mathrm{O}(\mathrm{An}=\mathrm{Ac}, \mathrm{Am}, \mathrm{Cm}, \mathrm{Es}, \mathrm{Lr})$ which were calculated using Dunning's (4s1p) and (9s4p1d)
cc-pVDZ basis set [25] for $\mathrm{H}$ and $\mathrm{O}$, respectively. For An (30s25p19d13f2g) basis sets were used which were reduced by one $\mathrm{d}$ function in the case of Am and augmented by one $p$ function for Es and Lr. The results of LPP and SPP HF as well as AE DHF [28] calculations for actinide-oxygen distances $R_{e}$ as well as for actinide-water binding energies $E_{\text {bond }}$ of $\mathrm{An}^{3+}-\mathrm{H}_{2} \mathrm{O}$ $(\mathrm{An}=\mathrm{Ac}-\mathrm{Lr})$ are listed in Table 1.

\subsubsection{Actinide-oxygen distance}

The actinide-oxygen distances $R_{e}$ for $\mathrm{An}^{3+}-\mathrm{H}_{2} \mathrm{O}(\mathrm{An}=$ Ac-Lr) from LPP and SPP HF calculations using aug-cc$\mathrm{pVTZ}$ basis sets for $\mathrm{H}$ and $\mathrm{O}$ as well as the five AE DHF distances [28] which were calculated using cc-pVDZ basis sets for $\mathrm{H}$ and $\mathrm{O}$ are shown in Fig. 1. As one can see the distances calculated using LPPs/SPPs decrease continuously with increasing nuclear charge from $2.46 \AA$ for $\mathrm{Ac}^{3+}-\mathrm{H}_{2} \mathrm{O}$ to $2.19 \AA$ for $\mathrm{Lr}^{3+}-\mathrm{H}_{2} \mathrm{O}$. This decrease by $0.27 \AA$ is related to the actinide contraction and it is equal to the value found by AE DHF calculations.

The LPP HF results are in good agreement with both SPP HF and AE DHF [28] reference data, i.e. the actinide-oxygen distances determined using LPPs are at most $0.03 \AA$ (1\%) longer than the corresponding SPP $\mathrm{HF}$ and AE DHF values. The mean absolute error (m.a.e.) and the mean relative error (m.r.e.) relating to the SPP data amount to $0.01 \AA$ and $0.6 \%$, respectively. As it was expected, the errors in the beginning of the actinide series are slightly larger than those of the heavier homologues. This is due to the fact that the $5 \mathrm{f}$ shell becomes more compact with increasing nuclear charge and the approximation of including the $5 \mathrm{f}$ orbitals in the PP core holds better in the second half of the row.

A comparison between the actinide-oxygen distances obtained by using different basis set sizes shows that these distances are only slightly affected by the change of basis sets, i.e. the actinide-oxygen distances become at most 0.01 and $0.02 \AA$ shorter when extending cc-pVDZ to aug-cc-pVTZ and aug-cc-pVQZ basis sets, respectively. This confirms former investigations of the basis set superposition error (BSSE) for lanthanide(III) monohydrates which show that the maximal change in lanthanide-oxygen distances amounts to $0.01 \AA$ extending cc-pVDZ to cc-pVTZ basis sets [28]. The largest differences between LPP and SPP/AE results are 0.03/0.04 for all basis set sizes.

\subsubsection{Actinide-water binding energy}

The actinide-water binding energies $E_{\text {bond }}$ for $\mathrm{An}^{3+}$ $\mathrm{H}_{2} \mathrm{O}(\mathrm{An}=\mathrm{Ac}-\mathrm{Lr})$ from LPP and SPP HF calculations 
Table 1 Actinide-oxygen distances $R_{e}$ (in $\AA$ ) and binding energies $\mathrm{E}_{\mathrm{bond}}$ (in eV) for $\mathrm{An}^{3+}-\mathrm{H}_{2} \mathrm{O}(\mathrm{An}=\mathrm{Ac}-\mathrm{Lr})$ from LPP/HF, SPP/HF, and AE/DHF [28] calculations using cc-pVDZ, aug-cc-pVTZ, and aug-cc-pVQZ basis sets for $\mathrm{H}$ and $\mathrm{O}$

\begin{tabular}{|c|c|c|c|c|c|c|c|c|c|c|c|c|c|c|}
\hline \multirow[b]{3}{*}{ An } & \multicolumn{7}{|c|}{$R_{e}$} & \multicolumn{7}{|c|}{$\mathrm{E}_{\text {bond }}$} \\
\hline & \multicolumn{3}{|c|}{ LPP } & \multicolumn{3}{|c|}{ SPP } & \multirow[t]{2}{*}{$\mathrm{AE}$} & \multicolumn{3}{|c|}{ LPP } & \multicolumn{3}{|c|}{ SPP } & \multirow[t]{2}{*}{$\mathrm{AE}$} \\
\hline & VDZ & VTZ & VQZ & VDZ & VTZ & VQZ & & VDZ & VTZ & VQZ & VDZ & VTZ & VQZ & \\
\hline Ac & 2.46 & 2.46 & 2.45 & 2.47 & 2.46 & 2.45 & 2.45 & 3.55 & 3.29 & 3.25 & 3.55 & 3.27 & 3.33 & 3.62 \\
\hline Th & 2.44 & 2.43 & 2.42 & 2.42 & 2.41 & 2.39 & & 3.65 & 3.39 & 3.35 & 3.79 & 3.52 & 3.57 & \\
\hline $\mathrm{Pa}$ & 2.41 & 2.41 & 2.40 & 2.39 & 2.38 & 2.38 & & 3.74 & 3.48 & 3.44 & 3.87 & 3.60 & 3.65 & \\
\hline $\mathrm{U}$ & 2.39 & 2.38 & 2.38 & 2.36 & 2.36 & 2.35 & & 3.83 & 3.57 & 3.53 & 3.94 & 3.68 & 3.73 & \\
\hline $\mathrm{Np}$ & 2.37 & 2.36 & 2.35 & 2.35 & 2.34 & 2.33 & & 3.92 & 3.66 & 3.62 & 4.02 & 3.76 & 3.81 & \\
\hline $\mathrm{Pu}$ & 2.35 & 2.34 & 2.33 & 2.32 & 2.32 & 2.31 & & 4.00 & 3.75 & 3.70 & 4.11 & 3.85 & 3.90 & \\
\hline $\mathrm{Am}$ & 2.33 & 2.32 & 2.31 & 2.31 & 2.30 & 2.29 & 2.29 & 4.08 & 3.82 & 3.79 & 4.18 & 3.92 & 3.96 & 4.30 \\
\hline $\mathrm{Cm}$ & 2.31 & 2.30 & 2.30 & 2.30 & 2.29 & 2.28 & 2.27 & 4.16 & 3.91 & 3.87 & 4.22 & 3.96 & 4.01 & 4.37 \\
\hline $\mathrm{Bk}$ & 2.29 & 2.28 & 2.28 & 2.28 & 2.27 & 2.26 & & 4.24 & 3.98 & 3.94 & 4.31 & 4.05 & 4.10 & \\
\hline $\mathrm{Cf}$ & 2.27 & 2.27 & 2.26 & 2.26 & 2.25 & 2.24 & & 4.32 & 4.06 & 4.02 & 4.40 & 4.15 & 4.20 & \\
\hline Es & 2.26 & 2.25 & 2.25 & 2.24 & 2.24 & 2.23 & 2.23 & 4.39 & 4.13 & 4.10 & 4.46 & 4.22 & 4.27 & 4.59 \\
\hline $\mathrm{Fm}$ & 2.24 & 2.24 & 2.23 & 2.23 & 2.22 & 2.21 & & 4.46 & 4.20 & 4.17 & 4.55 & 4.31 & 4.36 & \\
\hline $\mathrm{Md}$ & 2.22 & 2.22 & 2.22 & 2.22 & 2.21 & 2.20 & & 4.54 & 4.29 & 4.25 & 4.59 & 4.36 & 4.41 & \\
\hline No & 2.21 & 2.20 & 2.20 & 2.22 & 2.20 & 2.20 & & 4.61 & 4.36 & 4.32 & 4.63 & 4.40 & 4.44 & \\
\hline $\mathrm{Lr}$ & 2.19 & 2.19 & 2.19 & 2.20 & 2.19 & 2.19 & 2.18 & 4.69 & 4.43 & 4.40 & 4.70 & 4.46 & 4.51 & 4.85 \\
\hline
\end{tabular}

Basis sets: VDZ, VTZ: LPP (8s7p6d2f); SPP (14s13p10d8f)/[6s6p5d4f]; VQZ: LPP (8s7p6d2f1g); SPP (14s13p10d8f6g)/[6s6p5d4f3g]; AE: An (30s25p19d13f2g); H, O cc-pVDZ

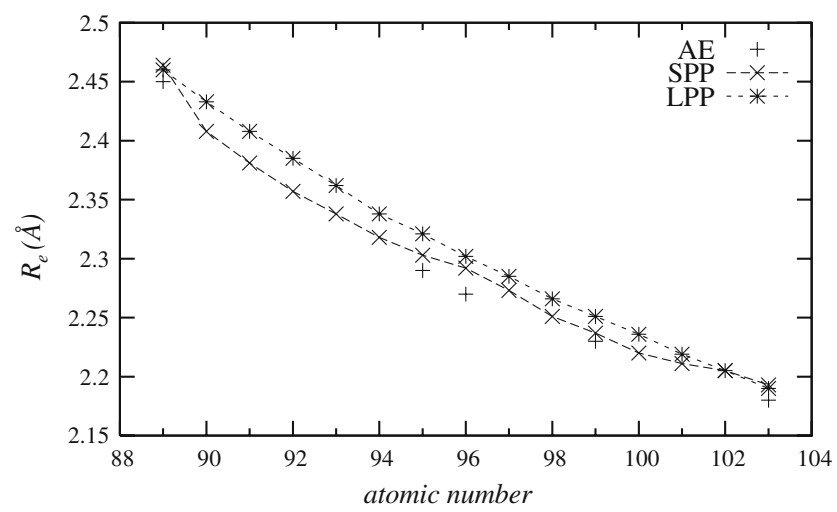

Fig. 1 Actinide-oxygen distances $R_{e}$ (in $\AA$ ) for $\mathrm{An}^{3+}-\mathrm{H}_{2} \mathrm{O}$ $(\mathrm{An}=\mathrm{Ac}-\mathrm{Lr})$ from LPP/HF, SPP/HF, and AE/DHF [28] calculations. Basis sets: LPP (8s7p6d2f); SPP (14s13p10d8f)/[6s6p5d4f]; AE (30s25p19d13f2g); H, O aug-cc-pVTZ for LPP and SPP and cc-pVDZ for AE calculations

using aug-cc-pVTZ basis sets for $\mathrm{H}$ and $\mathrm{O}$ as well as the five AE DHF binding energies [28] which were calculated using cc-pVDZ basis sets for $\mathrm{H}$ and $\mathrm{O}$ are shown in Fig. 2. As can be seen the binding energy increases by 1.14 and $1.19 \mathrm{eV}$ along the actinide series for LPP and SPP calculations, respectively. This is according to the decreasing actinide-oxygen distance which goes along with an increasing orbital overlap.

The binding energies calculated by using LPPs are in reasonable agreement with SPP reference data, i.e. the m.a.e. amounts to $0.08 \mathrm{eV}$ and the corresponding m.r.e. is $2 \%$. The largest deviation $(0.13 \mathrm{eV}(4 \%))$ occurs for

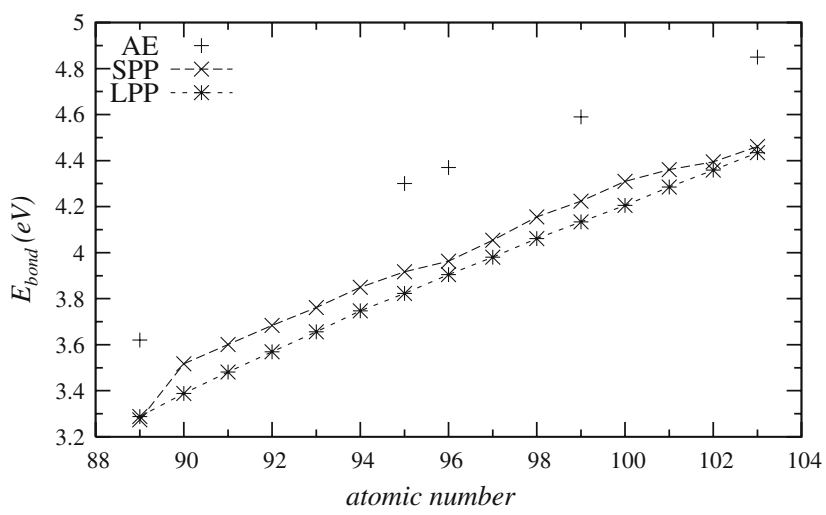

Fig. 2 Actinide-water binding energies $E_{\text {bond }}$ (in $\mathrm{eV}$ ) for $\mathrm{An}^{3+}$ $\mathrm{H}_{2} \mathrm{O}(\mathrm{An}=\mathrm{Ac}-\mathrm{Lr})$ from LPP/HF, SPP/HF, and AE/DHF [28] calculations. Basis sets: LPP (8s7p6d2f); SPP (14s13p10d8f)/ [6s6p5d4f]; AE (30s25p19d13f2g); H, O aug-cc-pVTZ for LPP and SPP and cc-pVDZ for AE calculations

Th. This can be explained by the fact that the difference between the $5 f$ occupation assumed for the LPP core and the $5 \mathrm{f}$ orbital population obtained by using the SPP is maximal for Th ( 0.08 electrons, cf. Table 2$)$.

As one can see from Fig. 2 there is a significant systematic disagreement between LPP HF and AE DHF [28] binding energies: m.a.e. and m.r.e. amount to $0.43 \mathrm{eV}$ and $10 \%$, respectively. This is due to the different basis sets applied for $\mathrm{H}$ and $\mathrm{O}$, i.e. aug-cc-pVTZ and cc$\mathrm{pVDZ}$ basis sets for $\mathrm{H}$ and $\mathrm{O}$ were used in LPP HF and AE DHF calculations, respectively. The comparison between LPP binding energies determined by using 
the same cc-pVDZ basis sets as applied for AE calculations shows much smaller deviations, i.e. m.a.e. and m.r.e. are $0.17 \mathrm{eV}$ and $4 \%$. The reason why these errors are still twice as large as those compared to SPP reference data is most likely the BSSE, which is typically smaller for PP than for AE calculations. At the AE DHF level a lower bound of $0.2 \mathrm{eV}$ for the BSSE in lanthanide-water binding energies of lanthanide(III) monohydrates can be extracted from the fact that the cc-pVDZ binding energies are higher than the cc-pVTZ values by up to this amount [28]. Unfortunately, corresponding values have not been determined for the actinide(III) monohydrates and estimates of the whole BSSE based on the counter-poise prescription of Boys and Bernardi have not been derived for lanthanides and actinides. However, from the comparison of atomic total AE DHF energies derived at the finite-difference and basis set level (cf. Table 1 in [28]) one may expect that the metal atom BSSE contribution for the actinide systems is significantly larger (up to a factor of 2) than for the lanthanide systems. Consequently actinide-water binding energies obtained by AE DHF calculations [28] are most likely stronger overestimated than those of PP calculations and deviations between AE DHF and LPP HF values are larger than those between SPP and LPP results.

A comparison between the actinide-water binding energies obtained by using different basis sets for $\mathrm{H}$ and $\mathrm{O}$ shows that these energies are strongly affected by BSSE, i.e. in agreement with the AE DHF results of Mochizuki and Tatewaki [28] the actinide-water bind- ing energies decrease by an average of $0.26 \mathrm{eV}(6 \%)$ by augmenting cc-pVDZ to aug-cc-pVTZ basis sets. Further increasing to aug-cc-pVQZ basis sets gives only by an average of $0.04 \mathrm{eV}(1 \%)$ lower binding energies so aug-cc-pVTZ basis sets seem to be large enough to describe the water ligand.

The differences between LPP and SPP binding energies are nearly the same using cc-pVDZ and aug-ccpVTZ basis sets, i.e. the m.a.e. amount to $0.08 \mathrm{eV}$ for both basis sets and the maximal errors are 0.14 and $0.13 \mathrm{eV}$ for cc-pVDZ and aug-cc-pVTZ basis sets, respectively. However, the deviations using aug-ccpVQZ basis sets are slightly larger (m.a.e.: $0.17 \mathrm{eV}$; maximal error: $0.22 \mathrm{eV}$ ) what is probably due to the greater number of $\mathrm{g}$ functions used in the SPP calculations (SPP: $(6 \mathrm{~g}) /[3 \mathrm{~g}]$; LPP: $(1 \mathrm{~g}))$.

\subsubsection{Mulliken orbital populations}

Table 2 shows the Mulliken orbital populations obtained by LPP and SPP HF calculations, respectively. It can be seen that the bonding between the trivalent metal ion $\mathrm{An}^{3+}$ and water is of the coordinate type where electrostatic attraction is the dominant factor stabilizing the system with dative contributions helping to a certain extent. The lone pairs of water act as electron donors and the valence $\mathrm{s}, \mathrm{p}$, and d orbitals of An behave as electron acceptors [28]. The valence $s$ and $p$ shells get slightly more occupied with increasing nuclear charge, e.g. the $7 \mathrm{~s} / 7 \mathrm{p}$ occupation increases from $0.03 / 0.01$ for $\mathrm{Ac}^{3+}-\mathrm{H}_{2} \mathrm{O}$ to $0.10 / 0.04$ for $\mathrm{Lr}^{3+}-\mathrm{H}_{2} \mathrm{O}$ in the case of

Table 2 Mulliken 6s, 6p, 6d, and 5f orbital populations and atomic charges (Q) on $\mathrm{An}$ in $\mathrm{An}^{3+}-\mathrm{H}_{2} \mathrm{O}(\mathrm{An}=\mathrm{Ac}-\mathrm{Lr})$ from LPP and SPP HF calculations

\begin{tabular}{|c|c|c|c|c|c|c|c|c|c|c|}
\hline \multirow[b]{2}{*}{ An } & \multicolumn{2}{|c|}{$\mathrm{s}$} & \multicolumn{2}{|c|}{$\mathrm{p}$} & \multicolumn{2}{|c|}{$\mathrm{d}$} & \multicolumn{2}{|c|}{$\mathrm{f}$} & \multicolumn{2}{|c|}{ Q } \\
\hline & LPP & SPP & LPP & SPP & LPP & SPP & $\mathrm{LPP}^{\mathrm{a}}$ & SPP & LPP & SPP \\
\hline Ac & 2.02 & 2.03 & 5.99 & 6.01 & 0.09 & 0.08 & 0.03 & 0.02 & 2.87 & 2.87 \\
\hline Th & 2.02 & 2.03 & 5.98 & 6.01 & 0.11 & 0.20 & 0.03 & 0.92 & 2.85 & 2.84 \\
\hline $\mathrm{Pa}$ & 2.02 & 2.03 & 5.99 & 6.01 & 0.12 & 0.11 & 0.03 & 2.01 & 2.85 & 2.84 \\
\hline $\mathrm{U}$ & 2.03 & 2.04 & 6.00 & 6.01 & 0.11 & 0.11 & 0.02 & 3.02 & 2.85 & 2.82 \\
\hline $\mathrm{Np}$ & 2.03 & 2.04 & 6.00 & 6.02 & 0.11 & 0.12 & 0.02 & 4.02 & 2.85 & 2.80 \\
\hline $\mathrm{Pu}$ & 2.03 & 2.05 & 6.00 & 6.02 & 0.11 & 0.13 & 0.02 & 5.03 & 2.84 & 2.77 \\
\hline $\mathrm{Am}$ & 2.03 & 2.06 & 6.00 & 6.03 & 0.11 & 0.14 & 0.02 & 6.03 & 2.84 & 2.75 \\
\hline $\mathrm{Cm}$ & 2.03 & 2.06 & 6.00 & 6.03 & 0.12 & 0.14 & 0.02 & 7.03 & 2.84 & 2.74 \\
\hline $\mathrm{Bk}$ & 2.03 & 2.07 & 6.00 & 6.03 & 0.12 & 0.14 & 0.02 & 8.03 & 2.84 & 2.73 \\
\hline $\mathrm{Cf}$ & 2.03 & 2.07 & 6.00 & 6.04 & 0.12 & 0.15 & 0.02 & 9.03 & 2.84 & 2.71 \\
\hline Es & 2.03 & 2.08 & 6.00 & 6.04 & 0.11 & 0.15 & 0.02 & 10.03 & 2.84 & 2.70 \\
\hline $\mathrm{Fm}$ & 2.03 & 2.08 & 6.00 & 6.04 & 0.12 & 0.15 & 0.02 & 11.03 & 2.83 & 2.69 \\
\hline $\mathrm{Md}$ & 2.03 & 2.09 & 6.00 & 6.04 & 0.12 & 0.15 & 0.02 & 12.03 & 2.83 & 2.69 \\
\hline No & 2.04 & 2.09 & 6.00 & 6.04 & 0.12 & 0.14 & 0.02 & 13.02 & 2.82 & 2.70 \\
\hline $\mathrm{Lr}$ & 2.04 & 2.10 & 6.00 & 6.04 & 0.12 & 0.14 & 0.02 & 14.02 & 2.82 & 2.69 \\
\hline
\end{tabular}

A $6 s^{2} 6 p^{6} 6 d^{1} 7 s^{2}$ ground state valence subconfiguration is considered for An. Basis sets: LPP (8s7p6d2f); SPP (14s13p10d8f)/[6s6p5d4f]; $\mathrm{H}, \mathrm{O}$ aug-cc-pVTZ

a $0-14$ electrons in the $5 f$ shell are attributed to the LPP core for Ac-Lr, respectively 
the SPP calculations. The valence $6 \mathrm{~d}$ occupation, however, stays relatively constant, i.e. an average of 0.11 and 0.14 electrons occupation for $6 \mathrm{~d}$ of all $\mathrm{An}^{3+}-\mathrm{H}_{2} \mathrm{O}$ are observed for LPP and SPP calculations, respectively. The increasing valence $s, p$ and the constant valence $d$ occupations can be attributed to increasing relativistic effects along the actinide series. The direct relativistic effects are dominating for $\mathrm{s}$ and $\mathrm{p}$ shells resulting in a stabilization and contraction of $7 \mathrm{~s} / 7 \mathrm{p}$, whereas for $\mathrm{d}$ shells the indirect relativistic effects are larger and lead to a destabilization and expansion of $6 \mathrm{~d}$. As one can see from SPP calculations the $5 \mathrm{f}^{n}$ occupation of the trivalent ion $\mathrm{An}^{3+}$ remains almost equal (maximal +0.03 electrons) by binding a water molecule except for Th $(0.92$ instead of 1.00 electron). Thus, the $5 \mathrm{f}$ orbitals do not participate significantly in chemical bonding of $\mathrm{An}^{3+}$ $\mathrm{H}_{2} \mathrm{O}$ and the $5 \mathrm{f}$-in-core approximation yields reasonable results for these complexes. Just for Th the $5 f$ population calculated using the SPP reveals a significant difference to the $5 \mathrm{f}$ occupation assumed for the LPP core wherefore the largest deviations occur for this element.

\subsection{Actinide trifluorides}

The HF calculations for $\mathrm{AnF}_{3}(\mathrm{An}=\mathrm{Ac}-\mathrm{Lr})$ using LPPs will be compared to corresponding SPP calculations. The comparison is reasonable for the second half, but critical for the first half of the actinide series. This is due to mixing of $\mathrm{f}$ and $\mathrm{s}$ orbitals of $\mathrm{AnF}_{3}(\mathrm{An}=\mathrm{Th}-\mathrm{Pu})$ in the case of the SPP calculations whereby the orbitals used for state-averaged MCSCF calculations are not pure f. Hence, all results will be compared separately for the first (Ac-Am) and second ( $\mathrm{Cm}-\mathrm{Lr})$ half of the actinide row. The results of both LPP and SPP calculations for actinide-fluorine bond lengths $R_{e}$ and angles $\angle \mathrm{F}-\mathrm{An}-\mathrm{F}$ as well as for actinide-fluorine binding energies $E_{\text {bond }}$ of $\mathrm{AnF}_{3}(\mathrm{An}=\mathrm{Ac}-\mathrm{Lr})$ are listed in Table 3.

\subsubsection{Actinide trifluorides structures}

The actinide-fluorine bond length decreases by 0.17 and $0.18 \AA$ along the actinide series for LPP and SPP HF calculations, respectively. Thus, the actinide contraction is by about $0.1 \AA$ smaller compared to those of $\mathrm{An}^{3+}-\mathrm{H}_{2} \mathrm{O}$ (0.27 $\AA$, cf. Sect. 3.1.1). The reason for this minor actinide contraction is that the An-F bond is more 'rigid' than the An-O coordination interaction in the water complexes [15,29]. This can be seen from the bond energies which are up to $1.96 \mathrm{eV}$ larger in the case of $\mathrm{AnF}_{3}$ (cf. Tables 1,3).

A comparison of actinide-fluorine bond lengths $R_{e}$ and angles $\angle \mathrm{F}-\mathrm{An}-\mathrm{F}$ of $\mathrm{AnF}_{3}(\mathrm{An}=\mathrm{Ac}-\mathrm{Lr})$ obtained using LPPs and SPPs demonstrates that the newly devel- oped LPPs yield quite accurate results for the whole actinide series. The m.a.e. for the first (second) half of the actinide row amount to $0.04(0.01) \AA$ and $5(3)^{\circ}$ for bond lengths and angles, respectively. The corresponding m.r.e. are $2(0.7) \%$ and $5(3) \%$. The largest errors in bond lengths occur for Th and $\mathrm{Pa}(0.07 \AA)$ because here the deviations between the assumed integral $5 \mathrm{f}$ occupation for the LPP core and the $5 \mathrm{f}$ population obtained using the SPP becomes maximal (Th 0.40 electrons, $\mathrm{Pa}$ 0.48 electrons, cf. Table 4$)$.

\subsubsection{Actinide-fluorine bond energy}

In contrast to the $\mathrm{An}^{3+}-\mathrm{H}_{2} \mathrm{O}$ complexation energy the actinide-fluorine binding energy of $\mathrm{AnF}_{3}$ decreases (by $0.48 \mathrm{eV}$ ) with increasing nuclear charge. This is most likely due to the decreasing actinide-fluorine bond length which is accompanied by an increasing repulsion between the fluorine atoms.

The differences in the actinide-fluorine binding energy between LPP and SPP calculations for the first half of the actinide series are obviously larger than for the second one, i.e. the m.a.e. and m.r.e. for the first (second) half are $0.13(0.05) \mathrm{eV}$ and 3 (1)\%, respectively. However, this is most likely due to a mixing of valence $f$ orbitals mainly with valence $s$ and $d$ orbitals in the SPP state-averaged MCSCF calculations especially of the elements Ac-Pa (cf. below). Once again, the largest errors are found for Th $(0.41 \mathrm{eV}, 7 \%)$ and $\mathrm{Pa}$ $(0.26 \mathrm{eV}, 5 \%)$ because they show the maximal discrepancies between the assumed LPP and the calculated SPP $5 \mathrm{f}$ population.

At this point one might ask how to calculate a binding energy with respect to the atoms in their experimentally observed ground states, e.g. at the correlated level. We propose to follow the strategy proposed for the lanthanide PPs almost two decades ago [7] and to calculate the binding energy with respect to the actinide atom in its lowest valence substate corresponding to the $5 \mathrm{f}^{n}$ subconfiguration, i.e. $5 \mathrm{f}^{n} 6 \mathrm{~d}^{1} 7 \mathrm{~s}^{2}$. The energy difference to the experimentally observed ground state, possibly belonging to a different configuration, can then be determined, e.g. at the AE DHF level [30], and corrected by electron correlation contributions to the energy difference (between the lowest levels) taken from experiment [13]. If desired, correlation contributions can of course also be obtained by $5 \mathrm{f}$-in-valence PP or AE calculations thus eliminating any empirical corrections.

\subsubsection{Mulliken orbital populations}

Mulliken orbital populations obtained by LPP and SPP HF calculations are listed in Table 4. As one can see the 
Table 3 Actinide-fluorine bond lengths $R_{e}$ (in $\AA$ ) and angles $\angle \mathrm{F}-\mathrm{An}-\mathrm{F}$ (in deg) as well as binding energies $\mathrm{E}_{\mathrm{bond}}$ (in $\mathrm{eV}$ ) for $\mathrm{AnF} 3$ $(\mathrm{An}=\mathrm{Ac}-\mathrm{Lr})$ from LPP and SPP HF calculations

\begin{tabular}{|c|c|c|c|c|c|c|}
\hline \multirow[b]{2}{*}{ An } & \multicolumn{2}{|c|}{$R_{e}$} & \multicolumn{2}{|c|}{$\angle \mathrm{F}-\mathrm{An}-\mathrm{F}$} & \multicolumn{2}{|c|}{$\mathrm{E}_{\text {bond }}$} \\
\hline & LPP & SPP & LPP & SPP & LPP & SPP \\
\hline Ac & 2.21 & 2.21 & 116 & 116 & 5.21 & 5.19 \\
\hline Th & 2.19 & 2.12 & 115 & 102 & 5.08 & 5.49 \\
\hline $\mathrm{Pa}$ & 2.18 & 2.11 & 115 & 114 & 5.00 & 5.25 \\
\hline $\mathrm{U}$ & 2.17 & 2.12 & 115 & 108 & 4.94 & 5.05 \\
\hline $\mathrm{Np}$ & 2.15 & 2.12 & 116 & 111 & 4.90 & 4.95 \\
\hline $\mathrm{Pu}$ & 2.14 & 2.11 & 116 & 112 & 4.86 & 4.86 \\
\hline $\mathrm{Am}$ & 2.13 & 2.10 & 117 & 113 & 4.84 & 4.77 \\
\hline $\mathrm{Cm}$ & 2.11 & 2.10 & 117 & 115 & 4.82 & 4.72 \\
\hline $\mathrm{Bk}$ & 2.10 & 2.08 & 118 & 115 & 4.80 & 4.72 \\
\hline $\mathrm{Cf}$ & 2.09 & 2.08 & 118 & 115 & 4.79 & 4.80 \\
\hline Es & 2.08 & 2.06 & 119 & 115 & 4.78 & 4.80 \\
\hline $\mathrm{Fm}$ & 2.07 & 2.05 & 119 & 114 & 4.76 & 4.79 \\
\hline $\mathrm{Md}$ & 2.06 & 2.04 & 119 & 116 & 4.76 & 4.82 \\
\hline No & 2.05 & 2.04 & 120 & 116 & 4.75 & 4.80 \\
\hline $\mathrm{Lr}$ & 2.04 & 2.03 & 120 & 117 & 4.73 & 4.77 \\
\hline
\end{tabular}

Basis sets: LPP (7s6p5d2f1g)/[5s4p3d2f1g]; SPP (14s13p10d8f6g)/[10s9p5d4f3g]; F aug-cc-pVQZ

Table 4 Mulliken 6s, 6p, 6d, and 5f orbital populations and atomic charges (Q) on An in $\mathrm{AnF}_{3}(\mathrm{An}=\mathrm{Ac}-\mathrm{Lr})$ from LPP and SPP HF calculations

\begin{tabular}{|c|c|c|c|c|c|c|c|c|c|c|}
\hline \multirow[b]{2}{*}{ An } & \multicolumn{2}{|c|}{$\mathrm{s}$} & \multicolumn{2}{|c|}{$\mathrm{p}$} & \multicolumn{2}{|c|}{ d } & \multicolumn{2}{|c|}{ f } & \multicolumn{2}{|c|}{ Q } \\
\hline & LPP & SPP & LPP & SPP & LPP & SPP & $\mathrm{LPP}^{\mathrm{a}}$ & SPP & LPP & SPP \\
\hline Ac & 2.00 & 2.03 & 6.00 & 6.03 & 0.31 & 0.39 & 0.13 & 0.23 & 2.56 & 2.29 \\
\hline Th & 2.00 & 2.10 & 5.99 & 6.07 & 0.33 & 0.85 & 0.10 & 0.60 & 2.57 & 2.37 \\
\hline $\mathrm{Pa}$ & 2.00 & 2.23 & 5.98 & 6.02 & 0.35 & 0.76 & 0.09 & 1.52 & 2.58 & 2.46 \\
\hline $\mathrm{U}$ & 2.00 & 1.96 & 5.98 & 5.98 & 0.36 & 0.48 & 0.08 & 3.02 & 2.58 & 2.56 \\
\hline $\mathrm{Np}$ & 2.00 & 1.97 & 5.97 & 5.97 & 0.36 & 0.48 & 0.07 & 4.05 & 2.59 & 2.53 \\
\hline $\mathrm{Pu}$ & 2.00 & 1.98 & 5.97 & 5.97 & 0.37 & 0.47 & 0.07 & 5.07 & 2.59 & 2.52 \\
\hline Am & 2.00 & 1.99 & 5.97 & 5.97 & 0.37 & 0.49 & 0.06 & 6.07 & 2.60 & 2.49 \\
\hline $\mathrm{Cm}$ & 2.00 & 2.00 & 5.97 & 5.97 & 0.37 & 0.47 & 0.06 & 7.07 & 2.60 & 2.50 \\
\hline $\mathrm{Bk}$ & 2.00 & 2.00 & 5.97 & 5.97 & 0.37 & 0.48 & 0.06 & 8.07 & 2.60 & 2.48 \\
\hline $\mathrm{Cf}$ & 2.00 & 2.01 & 5.97 & 5.99 & 0.36 & 0.46 & 0.05 & 9.07 & 2.61 & 2.47 \\
\hline Es & 2.01 & 2.01 & 5.97 & 5.99 & 0.36 & 0.48 & 0.05 & 10.07 & 2.61 & 2.44 \\
\hline $\mathrm{Fm}$ & 2.01 & 2.01 & 5.97 & 6.01 & 0.36 & 0.50 & 0.05 & 11.06 & 2.61 & 2.41 \\
\hline $\mathrm{Md}$ & 2.01 & 2.02 & 5.97 & 6.01 & 0.35 & 0.48 & 0.05 & 12.06 & 2.61 & 2.44 \\
\hline No & 2.01 & 2.03 & 5.98 & 6.01 & 0.35 & 0.46 & 0.05 & 13.05 & 2.61 & 2.45 \\
\hline $\mathrm{Lr}$ & 2.01 & 2.03 & 5.98 & 6.00 & 0.34 & 0.44 & 0.05 & 14.04 & 2.61 & 2.49 \\
\hline
\end{tabular}

A $6 s^{2} 6 \mathrm{p}^{6} 6 \mathrm{~d}^{1} 7 \mathrm{~s}^{2}$ ground state valence subconfiguration is considered for An. Basis sets: LPP (7s6p5d2f1g)/[5s4p3d2f1g]; SPP $(14 \mathrm{~s} 13 \mathrm{p} 10 \mathrm{~d} 8 \mathrm{f} 6 \mathrm{~g}) /[10 \mathrm{~s} 9 \mathrm{p} 5 \mathrm{~d} 4 \mathrm{f} 3 \mathrm{~g}] ;$ F aug-cc-pVQZ

a $0-14$ electrons in the $5 \mathrm{f}$ shell are attributed to the LPP core for Ac-Lr, respectively

bonding of $\mathrm{AnF}_{3}$ is of polar covalent nature. The three binding electron pairs are dragged more close to the fluorines end of the bonds, resulting in charge separations of up to 0.87 electrons per bond and a total atomic charge of up to 2.61 units on the actinide. Whereas the $s, p$, and f occupation numbers on the actinides are nearly integral, those of the d shells are not and point to some covalent contributions. The f orbital occupations determined using SPPs show that there is nearly no $5 \mathrm{f}$ orbital participation in the bonding of $\mathrm{AnF}_{3}$ except for Ac, Th, and $\mathrm{Pa}$, i.e. the $5 \mathrm{f}$ populations differ at most by 0.07 , $0.23,0.40$, and 0.48 electrons from the assumed $5 \mathrm{f} \mathrm{occu-}$ pations for the LPP core for $\mathrm{U}-\mathrm{Lr}, \mathrm{Ac}$, Th, and $\mathrm{Pa}$, respectively. At the AE WB level a low-lying $5 \mathrm{f}^{n-1} 6 \mathrm{~d}^{1}$ configuration is found for $\mathrm{Th}^{3+}$ and $\mathrm{Pa}^{3+}$ at only 0.50 and $1.62 \mathrm{eV}$, respectively, above the $5 \mathrm{f}^{n}$ configuration and explains the observed significant reduction of the $5 \mathrm{f}$ occupation number by configurational mixing in $\mathrm{ThF}_{3}$ and $\mathrm{PaF}_{3}$. This configurational mixing is also reflected by the higher $d$ population compared to the other $\mathrm{AnF}_{3}$. For all other actinide ions $\mathrm{An}^{3+}$ such configuration is at least $2.72 \mathrm{eV}$ higher and no significant configurational mixing is observed. Since the results for bond length, bond angle, and binding energy of $\mathrm{AcF}_{3}$ are in good 
agreement for LPP and SPP calculations (cf. Table 3), the f-part of the LPP is concluded to describe sufficiently the $5 \mathrm{f}$ participation in chemical bonding of these systems. This assumption is supported by the forbital population obtained by using LPPs which is the largest of the actinide series amounting to 0.13 electrons. Thus, the LPP $\mathrm{f}$ occupation including the assumed integral $5 \mathrm{f}$ occupation attributed to the PP core and the SPP $\mathrm{f}$ occupation of $\mathrm{AcF}_{3}$ differ only by 0.10 electrons. The LPPs are consequently a reasonable approximation for $\mathrm{AnF}_{3}$ except for $\mathrm{ThF}_{3}$ and $\mathrm{PaF}_{3}$.

\section{Conclusion}

Quasirelativistic energy-consistent 5f-in-core pseudopotentials corresponding to trivalent actinides $\left(5 \mathrm{f}^{n}\right.$ subconfiguration with $n=0-14$ for Ac-Lr) have been derived. Energy-optimized (6s5p4d), (7s6p5d), and (8s7p6d) GTO valence basis sets for use in molecular calculations as well as corresponding segmented contracted valence basis sets of VDZ, VTZ, and VQZ quality have been generated. Furthermore $2 \mathrm{f} 1 \mathrm{~g}$ polarization functions have been CI optimized. The deviations between energies obtained by numerical quasirelativistic AE HF calculations and corresponding values obtained by use of our pseudopotentials and basis sets are less than $0.2 \mathrm{eV}$. The differences using VTZ and VQZ contractions of $(7 \mathrm{~s} 6 \mathrm{p} 5 \mathrm{~d})$ and $(8 \mathrm{~s} 7 \mathrm{p} 6 \mathrm{~d})$ are at most $0.1 \mathrm{eV}$.

Results of HF test calculations on $\mathrm{An}^{3+}-\mathrm{H}_{2} \mathrm{O}$ and $\mathrm{AnF}_{3}(\mathrm{An}=\mathrm{Ac}-\mathrm{Lr})$ using the newly developed 5f-incore pseudopotentials show reasonable agreement with corresponding 5f-in-valence pseudopotential calculations even in the beginning of the actinide series, i.e. the m.a.e. (m.r.e.) for bond lengths amount to 0.01 $(0.6 \%)$ and $0.03 \AA(1 \%)$ and those for binding energies are $0.08(2 \%)$ and $0.09 \mathrm{eV}(2 \%)$ for $\mathrm{An}^{3+}-\mathrm{H}_{2} \mathrm{O}$ and $\mathrm{AnF}_{3}$, respectively. In the case of $\mathrm{An}^{3+}-\mathrm{H}_{2} \mathrm{O}$ the largest deviations occur for Th and in the case of $\mathrm{AnF}_{3}$ for Th and $\mathrm{Pa}$, since here the assumption of a near-integral $5 \mathrm{f}$ occupation is too crude. Besides these three cases the An $5 \mathrm{f}^{n}$ occupations in $\mathrm{An}^{3+}-\mathrm{H}_{2} \mathrm{O}$ and $\mathrm{AnF}_{3}$ deviate by at most 0.03 and 0.07 , respectively, from the integral occupation number in $\mathrm{An}^{3+}$. Thus, in these systems the $5 f$ orbitals do not participate significantly in chemical bonding and the 5f-in-core approximation yields quite accurate results at low computational cost.

Finally, we want to emphasize again that the derived 5f-in-core PPs will only lead to reasonable results for those cases where the An $5 f$ occupation number is close to integral. We recommend to explicitly test this condition, e.g. in single-point HF calculations with an explicit treatment of the $5 \mathrm{f}$ shell. In addition, for those cases where the 5 f occupation number deviates not too much from an integral value, the $5 \mathrm{f}$-in-core PPs might still be a useful computational tool, e.g. for preoptimizing structures of larger systems prior to more rigorous studies including the $5 \mathrm{f}$ shell explicitly.

\section{References}

1. Katz JJ, Seaborg GT, Morss LR (1986) The chemistry of the actinide elements. Chapman and Hall, London New York

2. Hoffman DC, Choppin GR (1986) J Chem Educ 63:1059

3. Pepper M, Bursten B (1991) Chem Rev 91:719

4. Schreckenbach G, Hay PJ, Martin RL (1999) J Comput Chem 20:70

5. Dolg M, Cao X (2003) The relativistic energy-consistent ab initio pseudopotential approach. In: Hirao K, Ishikawa Y (eds) Recent advances in relativistic molecular theory. World Scientific, New Jersey

6. Cao X, Dolg M (2006) Coord Chem Rev 250:900

7. Dolg M, Stoll H, Savin A, Preuss H (1989) Theor Chim Acta 75:173

8. Dolg M, Stoll H, Preuss H (1993) Theor Chim Acta 85:441

9. Dolg M, Stoll H, Preuss H (1989) J Chem Phys 90:1730

10. Cao X, Dolg M (2001) J Chem Phys 115:7348

11. Küchle W, Dolg M, Stoll H, Preuss H (1994) J Chem Phys 100:7535

12. Cao X, Dolg M, Stoll H (2003) J Chem Phys 118:487

13. Blaise J, Wyart J-F (1992) Energy levels and atomic spectra of actinides. In: International tables of selected constants, vol 20. CNRS, Paris

14. Field RW (1982) Ber Bunsenges Phys Chem 86:771

15. Dolg M, Stoll H (1996) Electronic structure calculations for molecules containing lanthanide atoms. In: Gschneidner Jr KA, Eyring L (eds) Handbook on the physics and chemistry of rare earths, vol 22. Elsevier, Amsterdam

16. Cao X, Dolg M (2003) Mol Phys 101:2427

17. Cao X, Li Q, Moritz A, Xie Z, Dolg M, Chen X, Fang W (2006) Inorg Chem 45:3444

18. Wiebke J, Moritz A, Cao X, Dolg M (2006) Phys Chem Chem Phys (accepted)

19. Froese Fischer C (1977) The Hartree-Fock method for atoms. Wiley, New York; program MCHF77, modified for pseudopotentials and quasirelativistic calculations by Dolg M (1995)

20. Yang J, Dolg M (2005) Theor Chem Acc 113:212

21. Pitzer RM (1979) Atomic electronic structure code ATMSCF. The Ohio State University, Columbus

22. Weigend F, Ahlrichs R (2005) Phys Chem Chem Phys 7:3297

23. Amos RD, Bernhardsson A, Berning A, Celani P, Cooper DL, Deegan MJO, Dobbyn AJ, Eckert F, Hampel C, Hetzer G, Knowles PJ, Korona T, Lindh R, Lloyd AW, McNicholas SJ, Manby FR, Meyer W, Mura ME, Nicklass A, Palmieri P, Pitzer R, Rauhut G, Schütz M, Schuhmann U, Stoll H, Tarroni AJSR, Thorsteinsson T, Werner HJ (2002) MOLPRO is a package of ab initio programs. University of Birmingham

24. http://www.theochem.uni-stuttgart.de/pseudopotentials

25. Dunning Jr TH (1989) J Chem Phys 90:1007

26. Kendall RA, Dunning Jr TH, Harrison RJ (1992) J Chem Phys 96:6769

27. Cao X, Dolg M (2004) J Molec Struct 673:203

28. Mochizuki Y, Tatewaki H (2001) Chem Phys 273:135

29. Wang SG, Schwarz WHE (1995) J Phys Chem 99:11687

30. Dyall KG, Grant IP, Johnson CT, Parpia FA, Plummer EP (1989) Comput Phys Commun 55:425; atomic structure code GRASP 